\title{
Outcomes in patients with lacrimal gland carcinoma treated with definitive radiotherapy or eye-sparing surgery followed by adjuvant radiotherapy
}

Yun-Hsuan Lin ${ }^{1,2,3 \dagger}$, Shih-Ming Huang ${ }^{3,4,5+}$, Wing-Keen Yap ${ }^{5}$, Ju-Wen Yang ${ }^{2,3}$, Ling Yeung ${ }^{2,3}$, Din-Li Tsan ${ }^{4}$, Joseph Tung-Chieh Chang ${ }^{5}$ and Lung-Chien Chen ${ }^{1 *}$

\begin{abstract}
Background: The optimal treatment for lacrimal gland cancer remains unclear. Eye-preserving surgery, as opposed to exenteration, followed by adjuvant radiotherapy (RT), has recently been reported to deliver satisfactory outcomes, but evidence is sparse. The aim of the present study was to evaluate outcomes in patients with lacrimal gland cancer treated at two tertiary medical centers.

Methods: We retrospectively examined data from patients with lacrimal gland cancer who had received eyepreserving surgical treatment followed by adjuvant RT with or without chemotherapy, or (if the tumor was inoperable) needle biopsy with definitive RT with or without chemotherapy. Baseline clinical and pathological characteristics were considered. Outcomes of interest included post-treatment complications, overall survival (OS), locoregional progression-free survival (LPFS), and distant metastasis-free survival (DMFS).

Results: Eighteen patients were included. Two-year OS, LPFS, and DMFS rates were 69.0, 76.7, and 71.4\%, respectively. Patients with early-stage (T1-T2) lacrimal gland cancer had significantly better outcomes than those with advanced-stage disease (T3-T4). Two-year OS, LPFS, and DMFS rates were each 100\% in patients with disease stages T1-T2, and 37.5, 50, and 37.5\%, respectively, in those with disease stages T3-T4 $(P<0.05)$. Orbital complications were well tolerated.

Conclusions: Eye-sparing surgery with adjuvant RT can achieve satisfactory results in patients with T1-T2 lacrimal gland carcinoma. Disease stage T3 and above was associated with poor outcomes even with post-operative RT, likely due to distant metastasis. Adding neoadjuvant chemotherapy or adjuvant chemotherapy to current treatment strategies might be a suitable choice for this group of patients.
\end{abstract}

Keywords: Lacrimal gland carcinoma, Orbital tumors, Radiotherapy, Orbital exenteration

\footnotetext{
* Correspondence: ocean@ntut.edu.tw

${ }^{\dagger}$ Yun-Hsuan Lin and Shih-Ming Huang contributed equally to this work.

'Department of Electro-Optical Engineering, National Taipei University of

Technology, 1, Sec. 3, Chung-Hsiao E. Rd, Taipei 10608, Taiwan, ROC

Full list of author information is available at the end of the article
}

(C) The Author(s). 2020 Open Access This article is licensed under a Creative Commons Attribution 4.0 International License, which permits use, sharing, adaptation, distribution and reproduction in any medium or format, as long as you give appropriate credit to the original author(s) and the source, provide a link to the Creative Commons licence, and indicate if changes were made. The images or other third party material in this article are included in the article's Creative Commons licence, unless indicated otherwise in a credit line to the material. If material is not included in the article's Creative Commons licence and your intended use is not permitted by statutory regulation or exceeds the permitted use, you will need to obtain permission directly from the copyright holder. To view a copy of this licence, visit http://creativecommons.org/licenses/by/4.0/ The Creative Commons Public Domain Dedication waiver (http://creativecommons.org/publicdomain/zero/1.0/) applies to the data made available in this article, unless otherwise stated in a credit line to the data. 


\section{Precis}

Eye-sparing surgery with adjuvant radiotherapy can achieve satisfactory results in patients with T1-2 lacrimal gland carcinoma. Disease stage T3 and above was associated with poor outcomes even with post-operative RT, likely due to distant metastasis.

\section{Introduction}

Lacrimal gland tumors are among the rarest types of head and neck cancers, accounting for approximately $10 \%$ of all orbital tumors [1]. Previous studies have estimated annual incidence for these tumors at $0.19-1$ per 1,000,000 people [1-3]. Epithelial lesions, including benign pleomorphic adenoma and malignant tumors, account for $20-30 \%$ of all lacrimal gland tumors. Malignant epithelial tumors include adenoid cystic carcinoma (ACC) (60\%), carcinoma ex pleomorphic adenoma or adenocarcinoma (20-30\%), and mucoepidermoid carcinoma (5\%) $[1,4,5]$. In contrast, non-epithelial lesions, such as inflammation, lymphoid tumors, plasmacytoma, histiocytoma, lipoma, and hemangioma account for $70-80 \%$ of all lacrimal gland tumors [2, 4, 6-8].

Lacrimal gland carcinomas are associated with poor local control and significant morbidity and mortality rates [9]. To date, no guidelines on standard treatment for lacrimal gland carcinomas have been developed due to the rarity of these diseases, which are conventionally treated with orbital exenteration followed by radiotherapy (RT) [5, 10]. However, locoregional recurrence, distant relapse, and cancer-related mortality risks are high after orbital exenteration [5, 11, 12]. Thus, eye-preserving surgery followed by adjuvant RT has recently gained popularity, but the optimal approaches to surgery and RT in these patients remain subject to debate $[5,9,13,14]$.

In this study, we examined clinical outcomes associated with either eye-preserving surgery followed by adjuvant RT, or needle biopsy followed by definitive $\mathrm{RT}$, in a cohort of 18 patients with lacrimal gland carcinoma. Clinical outcomes such as local control, survival, and ocular complications were considered. The clinical and pathological factors that affected the outcomes were also examined.

\section{Materials and methods}

This study protocol was reviewed and approved by the institutional review board of Chang Gung Memorial Hospital (Reference No.: 202000138B0). All data were stored in the hospital database and extracted for research. The participants' informed consent requirement was waived due to the retrospective nature of this study.

\section{Study population}

This retrospective study included patients with lacrimal gland cancer who underwent either eye-preserving surgical treatment followed by adjuvant RT with or without chemotherapy, or (in cases involving an inoperable tumor), needle biopsy followed by definitive RT with or without chemotherapy. Patients diagnosed with recurrent tumors at their first visit, and patients diagnosed with lacrimal duct cancer or other type of orbital cancer, were excluded. Disease stage was reviewed by a radiologist and ophthalmologist based on the American Joint Committee on Cancer (AJCC) staging system, eighth edition. All patient records included detailed pathological reports with data on cell type, margin status, perineural invasion (PNI), and lymphovascular invasion (LVSI), if the tumor had undergone resection.

\section{Treatment}

Radiotherapy was recommended for patients at high risk of recurrence, including those with an advanced stage lacrimal gland cancer and/or multiple unfavorable pathological factors such as bony structure invasion, positive surgical margins, LVSI, or PNI. RT was delivered in the form of three-dimensional conformal RT, intensity modulated RT, volumetric modulated arc therapy, or proton beam therapy. The RT field included the tumor bed and the residual gross tumor volume. The median prescribed dose for adjuvant RT was $50-60$ Gy and $66-70$ Gy for the definitive setting, with $1.8-2 \mathrm{~Gy}$ daily fractions administered over 5-7 weeks. Whether or not chemotherapy was given was at the attending physician's discretion. The regimens used were platinumbased chemotherapy.

\section{Statistical analyses}

Overall survival (OS) was defined as the time from the date of surgical treatment or biopsy until death or the last follow-up appointment. Local progression-free survival (LPFS) was defined as the time between the date of surgical treatment or biopsy and the detection of local tumor progression. Data on the first site(s) of distant metastasis were collected, and distant metastasis-free survival (DMFS) was determined. The Kaplan-Meier method was used to estimate the OS, LPFS, and DMFS rates, while the statistical significance of between-group differences in clinical characteristics (including sex, age, RT dose, cell types, and pathological features) and OS, LPFS, DMFS was determined using the log-rank test. All statistical analyses were performed using SPSS v. 23.0 (IBM Corp., New York, NY; formerly SPSS Inc., Chicago, IL). All $P$-values were two-sided, and a $P$-value $<0.05$ was considered statistically significant. 


\section{Post-therapy surveillance}

Follow-up in the form of radio-oncologic and ophthalmic clinic appointments was arranged every 3 months during the first 2 years, every 4-6 months during the third and fourth year, and every 6-12 months thereafter. Imaging examination was performed at specific intervals: chest radiography every 3 months, and computed tomography or orbital magnetic resonance imaging every 36 months, until symptoms indicating recurrence were noted.

\section{Ocular complication evaluation}

Follow-up in the form of ophthalmic clinic appointments was arranged every 3 months during the first 2 years and every 6-12 months thereafter. Detailed ocular examination, including visual acuity, intraocular pressure, slit lamp examination, and fundus examination was performed at every ophthalmologic visit. Medical or surgical treatment was administered if an ocular complication was noted.

\section{Results}

\section{Patient characteristics}

During 2000-2018, a total of 18 patients from the Linkou and Keelung branches of Chang Gung Memorial Hospital were included in the present study. The median follow-up time was 39 months (range 5-161 months) for the patients who were alive at the end of the study period, and 26 months (range 5-161 months) for the whole cohort. The patients' demographic and clinical characteristics are shown in Table 1 and Table 2. Fifteen patients $(83.3 \%)$ underwent eye-sparing surgery and the remaining patients had needle biopsy alone due to inoperable tumor invading brain tissue or extending to the infratemporal fossa. ACC was found in nine, carcinoma ex pleomorphic adenoma in seven, and poorly differentiated adenocarcinoma in two patients. In five patients (27.8\%), negative margins were achieved during surgery. Six patients (33.3\%) had pathologically confirmed LVSI and eight patients $(44.4 \%)$ had pathologically confirmed PNI. Patients who underwent eye-sparing surgery also received adjuvant RT or concurrent platinum-based chemoradiotherapy (CCRT) due to the presence of risk factors, such as positive surgical margin, confirmed LVSI or PNI, or initial advanced tumor size and invasion status. Three patients who underwent needle biopsy received definitive RT or CCRT, also platinum-based.

Regarding RT technique, 2 patients received threedimensional conformal radiotherapy (3DCRT), 14 received intensity-modulated radiotherapy (IMRT), one received proton beam therapy alone, and one received combined proton-photon RT. The dosimetry comparison between photon plan and proton plan is presented in Fig. 1. The median RT dose for adjuvant
RT was 6000 cGy (range 3600-7000 cGy) in 1.8-2 Gy per fraction, and the definitive RT dose was 6000 cGy/25 fractions, $7200 \mathrm{cGy} / 36$ fractions, and 7200 cGy/36 fractions for three patients, respectively.

\section{Survival analysis}

For the entire cohort, 2-year OS, LPFS, and DMFS rates were $69.0,76.7$, and $71.4 \%$, respectively (Fig. 2). Associations between clinicopathological factors and 2-year OS, LPFS, and DMFS rates are shown in Table 3. Patients with early-stage (T1-T2) lacrimal cancer achieved significantly better 2-year OS, LPFS, and DMFS than those with advanced-stage disease (T3T4). Two-year OS was $100 \%$ in patients with disease stage $\mathrm{T} 1-\mathrm{T} 2$, and $37.5 \%$ in those with disease stage T3-T4. Moreover, 2-year LPFS was $100 \%$ in patients with disease stage $\mathrm{T} 1-\mathrm{T} 2$ and $50 \%$ in those with disease stage T3-T4. Finally, 2-year DMFS was $100 \%$ in patients with disease stage $\mathrm{T} 1-\mathrm{T} 2$ and $37.5 \%$ in those with disease stage T3-T4 (Fig. 3). Other factors, including sex, age $(<54$ yrs. vs. $\geq 54$ yrs $)$, RT dose $(<66$ vs. $\geq 66$ Gy), cell types (ACC vs. others), margin (positive vs. negative), PNI (positive vs. negative), and LVSI (positive vs. negative) had no significant impact on OS, LPFS, or DMFS.

\section{Ocular toxicity analysis}

In our study subjects, one patient was lost follow up at the ophthalmic clinic 3 months after surgery and three patients 6 months after surgery. During their follow-up period, no ocular complications were reported. In the remaining 14 patients who were followed up by the ophthalmologist for more than 12 months, the administered treatment was well tolerated. Treatment-related acute and late ocular toxicity effects are shown in Table 4. Ocular toxicities, including punctate keratitis, developed in five patients (27.78\%) 1.14-7.9 months after radiotherapy (RT total/fraction dose: 5000-7000/200 cGy). Cataract developed in four patients $(22.22 \%)$ and occurred 2.13-112.7 months after the RT (RT total/fraction dose: 6000-6600/200 cGy). Dry eye developed in two patients (11.11\%) and occurred 6.2911.05 months after the RT (RT total/fraction dose: $6600 / 200$ cGy). Blepharitits, which developed in two patients (11.11\%), occurred 2.06-8.00 months after the treatment (RT total/fraction dose: 6600/200 cGy). Other complications, including trichiasis, acute conjunctivitis, corneal epithelial defect, filamentary keratitis, radiation retinopathy, and vitreous hemorrhage were each reported in one patient (5.56\%). The patient with radiation retinopathy received an intravitreous injection of $1.25 \mathrm{mg}$ Avastin (bevacizumab) for retinal neovascularization. 
Table 1 Baseline characteristics of patients with lacrimal gland carcinoma

\begin{tabular}{|c|c|c|}
\hline Total $(n=18)$ & Patient number & Percentage (\%) \\
\hline Median age (years) & $54(20-85)$ & \\
\hline \multicolumn{3}{|l|}{ Sex } \\
\hline Male & 8 & 44.4 \\
\hline Female & 10 & 55.6 \\
\hline \multicolumn{3}{|l|}{ T stage } \\
\hline 1 & 1 & 5.6 \\
\hline 2 & 9 & 50.0 \\
\hline 3 & 3 & 16.7 \\
\hline 4 & 5 & 27.7 \\
\hline \multicolumn{3}{|l|}{ Types of surgery } \\
\hline Excision & 15 & 83.3 \\
\hline Needle biopsy & 3 & 16.7 \\
\hline \multicolumn{3}{|l|}{ RT treatment } \\
\hline Adjuvant RT alone & 13 & 72.2 \\
\hline Adjuvant CCRT & 2 & 11.1 \\
\hline Definitive RT & 2 & 11.1 \\
\hline Definitive CCRT & 1 & 5.6 \\
\hline \multicolumn{3}{|l|}{ RT type } \\
\hline 3DCRT & 2 & 11.1 \\
\hline IMRT & 14 & 77.8 \\
\hline Proton & 2 & 11.1 \\
\hline \multicolumn{3}{|l|}{ RT dose } \\
\hline$<6600$ cGy & 9 & 50.0 \\
\hline$\geq 6600$ cGy & 9 & 50.0 \\
\hline \multicolumn{3}{|l|}{ Cell type } \\
\hline Adenoid cystic carcinoma & 9 & 50.0 \\
\hline Carcinoma ex pleomorphic adenoma & 7 & 38.9 \\
\hline Poorly differentiated adenocarcinoma & 2 & 11.1 \\
\hline \multicolumn{3}{|l|}{ Margin } \\
\hline positive & 13 & 72.2 \\
\hline negative & 5 & 27.8 \\
\hline \multicolumn{3}{|l|}{ LVSI } \\
\hline Positive & 6 & 33.3 \\
\hline Negative & 12 & 66.7 \\
\hline \multicolumn{3}{|l|}{ PNI } \\
\hline Positive & 8 & 44.4 \\
\hline Negative & 10 & 55.6 \\
\hline
\end{tabular}

RT radiotherapy, CCRT concurrent chemoradiotherapy, 3DCRT 3-dimensional conformal radiotherapy, IMRT intensity-modulated radiotherapy, CGy centigray, LVSI lymphovascular invasion, $P N /$ perineural invasion.

\section{Discussion}

This study reported outcomes in patients with lacrimal gland carcinoma who underwent either needle biopsy followed by definitive radiotherapy with or without chemotherapy due to inoperable tumor, or eye-preserving surgical treatment followed by adjuvant radiotherapy with or without chemotherapy. In the present cohort, 2-year OS, LPFS, and DMFS rates were 69.0, 76.7, and 71.4\%, respectively. These outcomes were inferior to those reported in previously, which was probably due to differences in patient 


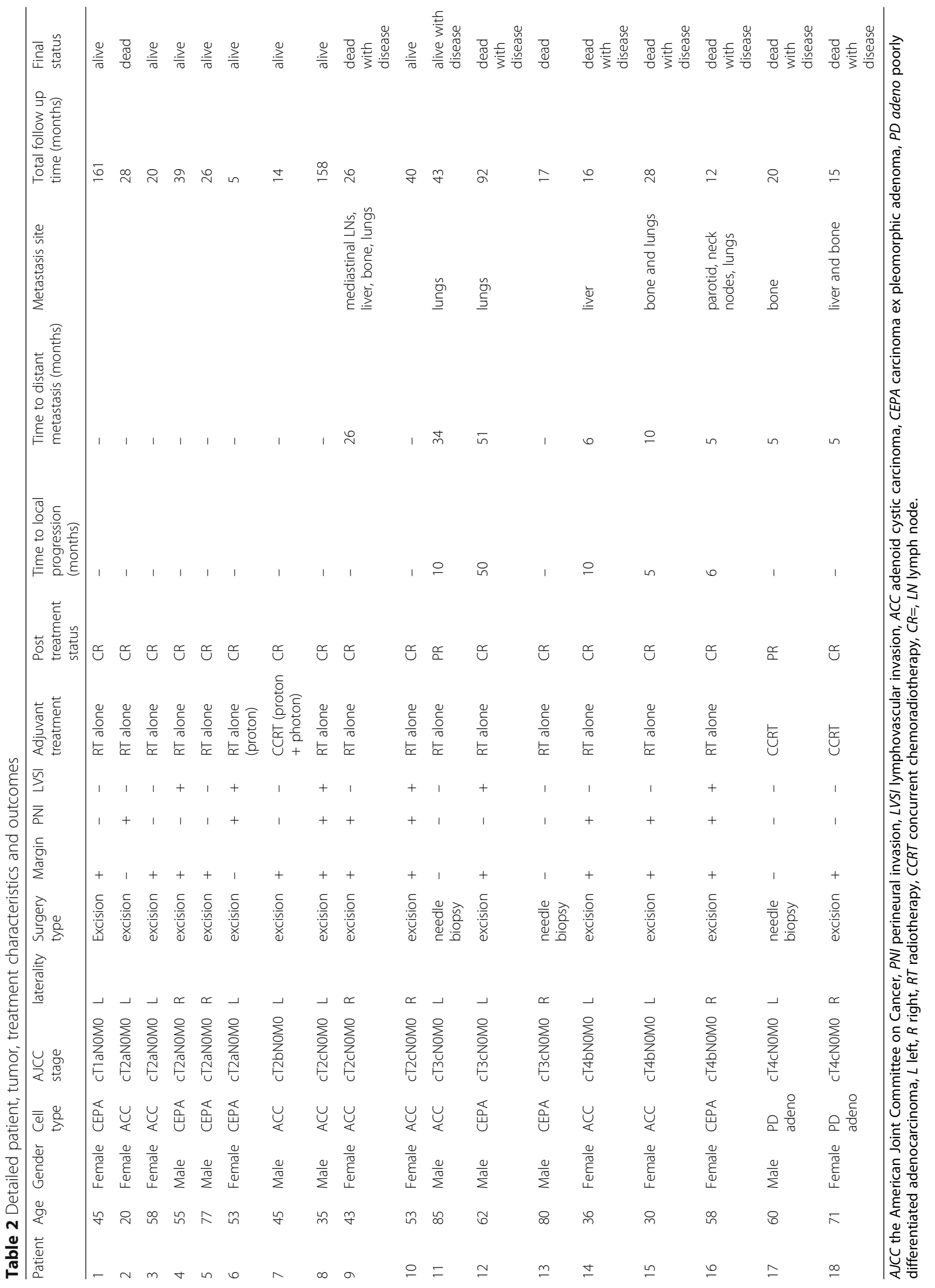



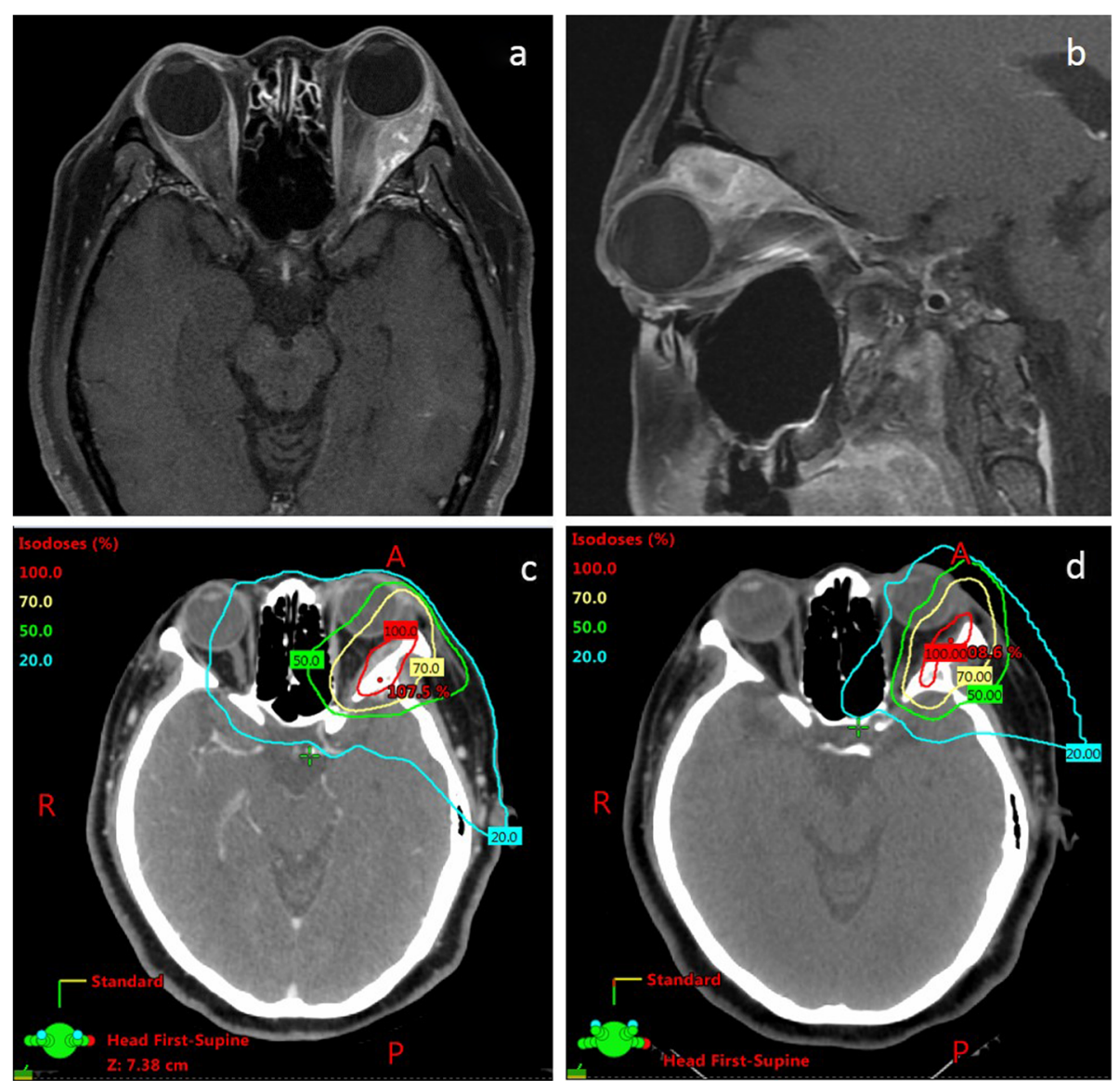

Fig. 1 Patient number 7, cT2bNOMO lacrimal gland cancer, pre-surgery T1-MRI and the photon or proton beam dosimetry of adjuvant radiotherapy. Total RT dose was $70 \mathrm{~Gy}$ in 33 fractions. Dose constraints for organs at risk: Brain stem: $\mathrm{D}_{\max }<54 \mathrm{~Gy}$. Left eye and left optic nerve: $D_{\max } \leq 59.5 \mathrm{~Gy}$ (85\% dose). Right eye and right optic nerve: $\mathrm{D}_{\max } \leq 50 \mathrm{~Gy}$. Optic chiasm: $\mathrm{D}_{\max }<54$ Gy. Right len: $\mathrm{D}_{\max } \leq 18 \mathrm{~Gy}$. No ocular complication was noted during our study period. a. Axial view of the tumor. b. Sagittal view of the tumor. c. Photon beam dosimetry. $1 \mathrm{~d}$. Proton beam dosimetry

characteristics. Esmaeli et al. [15] studied the eye-sparing approach to treating lacrimal gland carcinoma in $11 \mathrm{pa}-$ tients during 2007-2014. All 11 patients were disease-free at the last follow-up visit after the eye-sparing surgery, with a median follow-up time of 33 months (range, 14-64 months). A significant proportion of patients recruited by Esmaeli et al. had early-stage disease (one patient with T1, six patients with $\mathrm{T} 2$, one with $\mathrm{T} 3$, and three with T4). In addition, Han et al. [16] reported outcomes following eye-sparing surgery and adjuvant radiotherapy in 10 patients with adenoid cystic carcinoma of the lacrimal gland, treated during 1998-2012 (one patient with $\mathrm{T} 1$, seven with $\mathrm{T} 2$, and two with $\mathrm{T} 3$ stage disease), at a median follow-up time of 89.5 months (range 37-217 months). Reported OS was 90\%, with a single patient who died 58 months after surgery. Moreover, DMFS was 100\%, while local recurrence occurred in one patient. In our series, almost half of the patients had advanced-stage lacrimal gland carcinoma (three patients with T3 [16.7\%], and five patients with T4 [27.7\%]). In addition, the majority of patients were at high risk of locoregional or distant recurrence, with a positive surgical margin in $72.2 \%$ of the patients, and confirmed LVSI and PNI in 33.3 and $44.4 \%$ of patients, respectively. The differences in patient baseline characteristics may explain the relatively lower OS, LPFS, and DMFS rates reported in our study.

The present study is among a small number that have examined factors affecting outcomes in patients with lacrimal gland carcinoma, which is an aim that is difficult to achieve due to the rarity of this disease. In a previous report by Woo et al., the only tumor characteristic associated with outcome was $\mathrm{T}$ stage. In the same study, adjuvant radiotherapy was strongly associated with a better 5-year recurrence-free survival rate [17]. Friedrich and Bleckmann also found that lower $\mathrm{T}$ stage was associated with a better 


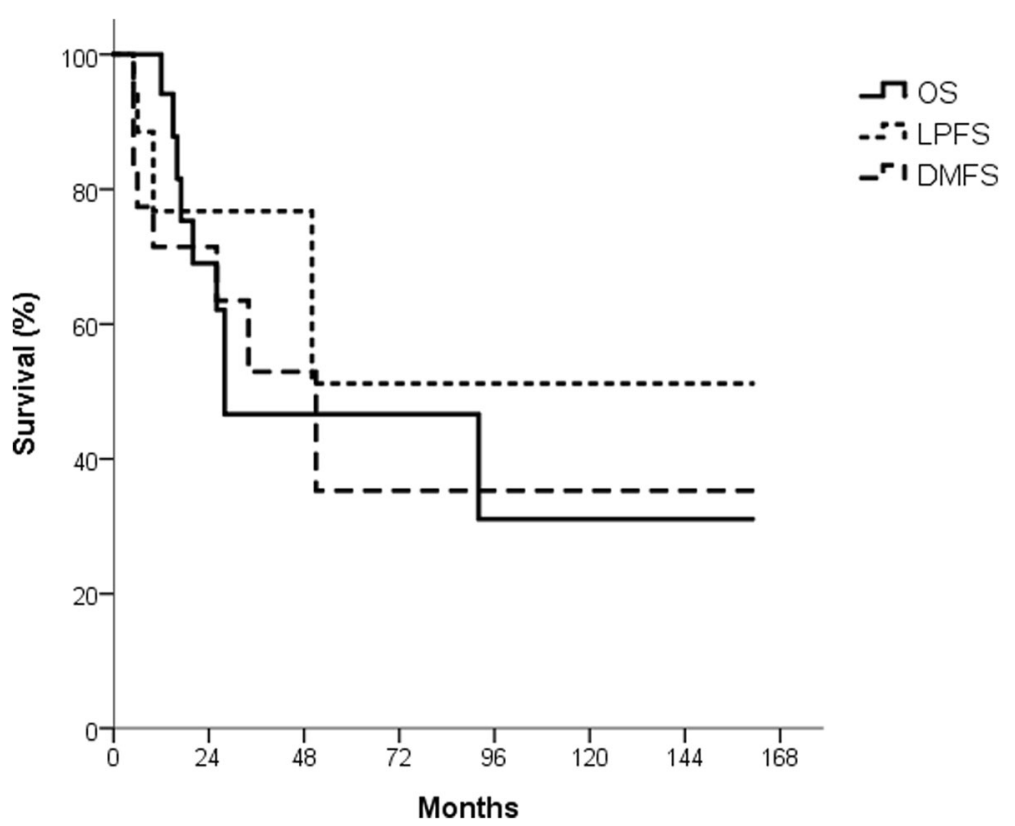

Fig. 2 The overall survival (OS), local progression-free survival (LPFS), and distant metastasis-free survival (DMFS) graphs of the whole cohort. For the entire cohort, 2-year OS, LPFS, and DMFS rates were 69.0, 76.7, and 71.4\%, respectively

prognosis [18]. Among factors reported as negatively affecting patient outcomes were older age, basaloid or solid pattern ACC, perineural invasion, and the presence of a macroscopic tumor on imaging scans before RT. [12, 19-22] In the present study, among patients who had received adjuvant radiotherapy, $\mathrm{T}$ stage and tumor size emerged as the most important factors that determined patient outcome. Specifically, patients with tumor at stage T1-2 treated with eye-sparing surgery followed by adjuvant radiotherapy achieved $100 \%$ 2-year LPFS, DMFS, and OS rates. However, tumor stage T3 and higher was associated with poor outcomes even when treated with surgical resection followed by adjuvant RT, which occurred most likely due to distant metastasis. Adding neoadjuvant chemotherapy or adjuvant chemotherapy to current treatment strategies might be a suitable choice for this group of patients to improve the control of distant disease.

Proton therapy or even heavy ion therapy applied to lacrimal gland cancer is considered promising for reducing low dose delivered outside the treated fields [3]. We have observed better dosimetry associated with the proton plan compared to the photon plan.

Table 3 Two-year overall survival, local progression-free survival, and distant-metastasis free survival rates for patients with lacrimal gland carcinoma, including factors affecting survival

\begin{tabular}{lllllll}
\hline & 2-Y OS (\%) & $P$-value* & 2-Y LPFS (\%) & $P$-value* & 2-Y DMFS (\%) & $P$-value* \\
\hline Sex (male vs. female) & 87.5 vs. 67.5 & 0.533 & 87.5 vs. 67.5 & 0.533 & 87.5 vs. 57.1 & 0.353 \\
Age (<54 vs. $\geqq 54)$ & 85.7 vs. 55.6 & 0.529 & 76.2 vs. 77.8 & 0.621 & 75.0 vs. 66.7 & 0.331 \\
T stage (T1-2 vs. T3-4) & 100.0 vs. 37.5 & 0.013 & 100.0 vs.50.0 & 0.006 & 100.0 vs. 37.5 & 0.002 \\
RT dose (<66 vs. 66 Gy) & 87.5 vs. 50.8 & 0.114 & 63.5 vs. 88.9 & 0.464 & 75.0 vs. 66.7 & 0.344 \\
Cell types (ACC vs. others) & 87.5 vs. 50.0 & 0.517 & 66.7 vs. 87.5 & 0.581 & 77.8 vs. 66.7 & 0.914 \\
Margin (positive vs. negative) & 75.5 vs. 50.0 & 0.43 & 76.9 vs. 75.0 & 0.944 & 69.2 vs. 80.0 & 0.76 \\
PNI (positive vs. negative) & 66.7 vs. 71.4 & 0.438 & 58.3 vs. 90.0 & 0.264 & 58.3 vs. 80.0 & 0.583 \\
LVSI (positive vs. negative) & 80.0 vs. 75.0 & 0.879 & 80.0 vs. 75.0 & 0.879 & 83.3 vs. 66.7 & 0.292 \\
\hline
\end{tabular}

*Log-rank test

$Y$ year, OS overall survival, LPFS local progression free survival, DMFS distant-metastasis free survival, $R T$ radiotherapy, $A C C$ adenoid cystic carcinoma, $P N I$ perineural invasion, LVSI lymphovascular invasion 


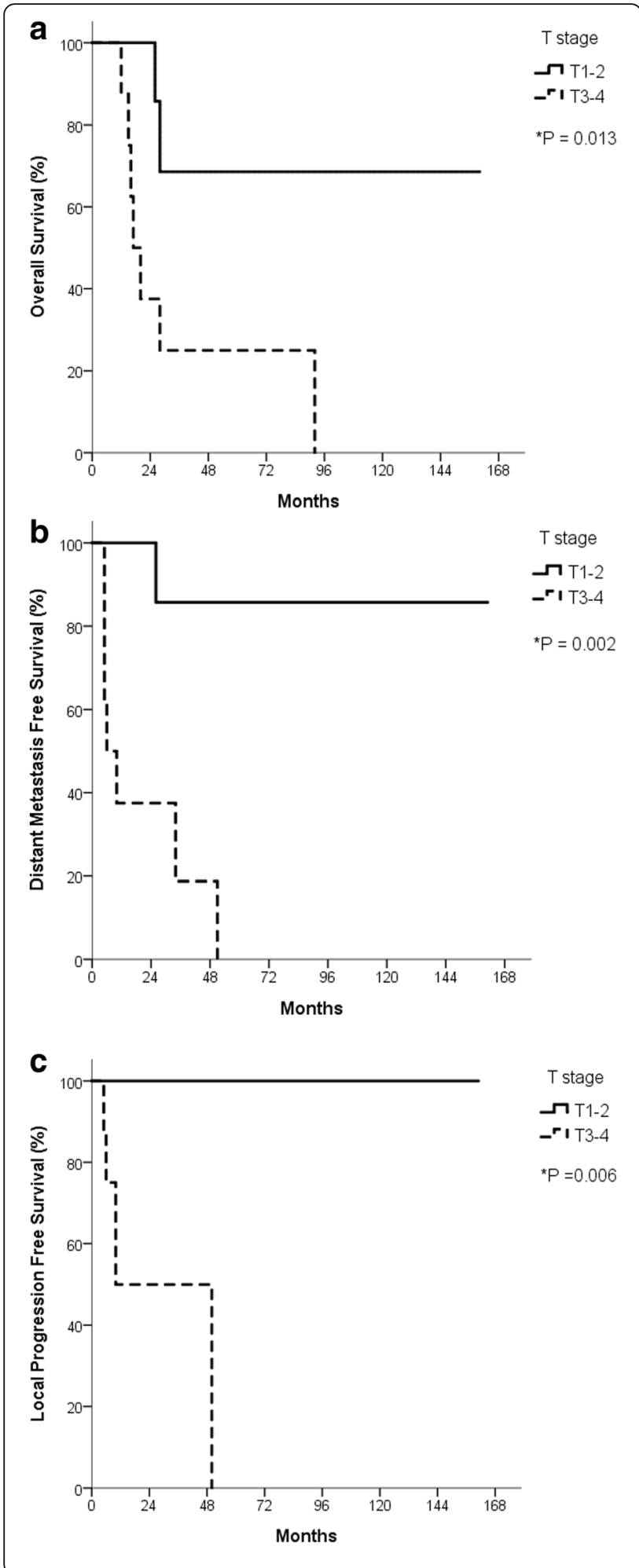

Fig. 3 Kaplan-Meier survival curves showing overall survival (a), local progression free survival (b), and distant-metastasis free survival (c) in patients with lacrimal gland carcinoma according to $T$ category as determined using the eighth edition of the American Joint Committee on Cancer staging system. ${ }^{*}$ Log-rank test. (a) The 2-year OS rates were 100 and $37.5 \%$ in T1-2 and T3-4 groups ( $p=0.013$ ), with (b) the 2-year DMFS rates were 100 and $37.5 \%(p=0.002)$, and (c) the 2-year LPFS rates of 100 and $50 \%$, respectively $(p=0.006)$

At a high dose ( $>70 \%$ dose), proton beam dosimetry is equivalent to photon therapy; however, low-dose distribution is better for proton therapy, whereby the $20 \%$ isodose line can spare the opposite eye. Ensuring low dose spread to the nearby healthy tissue is critical in certain cases. Lower dose delivered to the regional organ at risk can reduce the likelihood of secondary malignancy and long-term complications, and therefore should be considered for patients with early-stage lacrimal gland cancer who are likely to achieve good local control and long-term survival after the treatment.

Several ocular toxicities were recorded among our patients. These complications were expected as the radiation doses used were mostly above the dose that is well tolerated by ocular structures other than the sclera. Sensitivity to radiation of the ocular structures has been shown to vary [23-25]. The lens has been reported as the most radiosensitive ocular structure, with a minimum cataractogenic dose of $5.5 \mathrm{~Gy}$. The lacrimal gland, cornea, and conjunctiva can each tolerate up to $50 \mathrm{~Gy}$ of radiation. The retina tolerates doses $>55 \mathrm{~Gy}$. The sclera is the most resistant ocular structure and is believed to tolerate doses $>1000 \mathrm{~Gy}$. The majority of ocular complications reported in the present study were effectively managed by ophthalmologists. Only one patient received a punctal plug due to intolerable dry eye and another patient required intravitreous injection of Avastin (bevacizumab) at $1.25 \mathrm{mg}$ due to radiation-associated retinopathy with retinal neovascularization. Therefore, we recommend that all patients who have received RT for lacrimal gland carcinoma visit an ophthalmologic clinic regularly.

This study has some limitations. First, this was a retrospective study based on medical records, which makes selection bias inevitable. Moreover, some of the patients did not attend the ophthalmologic clinic as recommended, making it likely that the rate of ocular complications was underestimated. Second, due to the rarity of lacrimal gland cancer, the small sample size meant that the analysis was unlikely to yield statistically significant results. Finally, the patients receiving proton treatment were only followed for 1-2 years, as our hospital began proton beam therapy in 2017. A longer follow-up period 
Table 4 Frequency of ocular complications after radiotherapy reported in the current study and the corresponding radiotherapy dose

\begin{tabular}{llllll}
\hline Complications & Patient number & Percentage (\%) & Time of occurrence $(\mathrm{m})$ & RT total dose (cGy) & RT fraction dose (cGy) \\
\hline Punctate keratitis & 5 & 27.78 & $1.14-7.9$ & $5000-7000$ & 200 \\
Cataract & 4 & 22.22 & $2.13-112.7$ & $6000-6600$ & 200 \\
Dry eye & 2 & 11.11 & $6.49-11.05$ & 6600 & 200 \\
Blepharitis & 2 & 11.11 & $2.06-8.00$ & 6000 & 200 \\
Trichiasis & 1 & 5.56 & 16.36 & 6000 & 200 \\
Acute conjunctivitis & 1 & 5.56 & 0.68 & 5000 & 250 \\
Corneal epithelial defect & 1 & 5.56 & 23.38 & 7200 & 200 \\
Filamentary keratitis & 1 & 5.56 & 6.65 & 7000 & 200 \\
Radiation retinopathy & 1 & 5.56 & 74.39 & 6600 & 6840 \\
Vitreous hemorrhage & 1 & 5.56 & 10.92 & & 200 \\
\hline
\end{tabular}

$R T$ radiotherapy, cGy centigray

is required to evaluate the effects of proton therapy on patient survival.

In conclusion, eye-sparing surgery with adjuvant RT can achieve satisfactory results in patients with T1-2 lacrimal gland carcinoma. Disease stage T3 and above was associated with poor outcomes even with postoperative RT, probably due to distant metastasis. Adding neoadjuvant or adjuvant chemotherapy to current treatment strategies may be a suitable choice for this group of patients.

\section{Acknowledgements}

Not applicable.

\section{Authors' contributions}

SMH, YHL and LCC conceived the study; SMH and YHL collected data; SMH analyzed and interpreted data; YHL, SMH and WKY wrote manuscript; LCC provided statistical analyses; JWY, LY, DLT, and JTCC provided critical review and revision of manuscript; All authors reviewed the results and approved the final version of the manuscript.

\section{Funding}

The authors state that this work has not received any funding.

\section{Availability of data and materials}

The datasets used during the current study are available from the corresponding author on reasonable request.

\section{Ethics approval and consent to participate}

This study protocol was reviewed and approved by the institutional review board of Chang Gung Memorial Hospital (Reference No.: 202000138B0).

\section{Consent for publication}

All data were stored in the hospital database and extracted for research. The participants' informed consent requirement was waived due to the retrospective nature of this study.

\section{Competing interests}

The authors declare that they have no competing interests.

\section{Author details}

'Department of Electro-Optical Engineering, National Taipei University of Technology, 1, Sec. 3, Chung-Hsiao E. Rd, Taipei 10608, Taiwan, ROC. ${ }^{2}$ Department of Ophthalmology, Chang Gung Memorial Hospital, Keelung, Taiwan. ${ }^{3}$ College of Medicine, Chang Gung University, Taoyuan, Taiwan. ${ }^{4}$ Department of Radiation Oncology, Chang Gung Memorial Hospital,
Keelung, Taiwan. ${ }^{5}$ Department of Radiation Oncology, Chang Gung Memorial Hospital, Linkou, Taiwan

Received: 1 May 2020 Accepted: 16 June 2020

Published online: 22 June 2020

\section{References}

1. Shields JA, Shields CL, Epstein JA, Scartozzi R, Eagle RC Jr. Review: primary epithelial malignancies of the lacrimal gland: the 2003 Ramon L. font lecture. Ophthalmic Plast Reconstr Surg. 2004;20(1):10-21.

2. von Holstein SL, Therkildsen MH, Prause JU, Stenman G, Siersma VD, Heegaard S. Lacrimal gland lesions in Denmark between 1974 and 2007. Acta Ophthalmol. 2013;91(4):349-54.

3. Hayashi K, Koto M, Ikawa H, Ogawa K, Kamada T. Efficacy and safety of carbon-ion radiotherapy for lacrimal gland carcinomas with extraorbital extension: a retrospective cohort study. Oncotarget. 2018;9(16):12932-40.

4. $\mathrm{Ni}$ C, Kuo PK, Dryja TP. Histopathological classification of 272 primary epithelial tumors of the lacrimal gland. Chin Med J. 1992;105(6):481-5.

5. Wright JE, Rose GE, Garner A. Primary malignant neoplasms of the lacrimal gland. Br J Ophthalmol. 1992;76(7):401-7.

6. Alkatan HM, Al-Harkan DH, Al-Mutlaq M, Maktabi A, Elkhamary SM. Epithelial lacrimal gland tumors: a comprehensive clinicopathologic review of 26 lesions with radiologic correlation. Saudi J Ophthalmol. 2014;28(1):49-57.

7. Gao Y, Moonis G, Cunnane ME, Eisenberg RL. Lacrimal gland masses. AJR Am J Roentgenol. 2013:201(3):W371-81.

8. Zeng J, Shi JT, Li B, Sun XL, An YZ, Li LQ, et al. Epithelial tumors of the lacrimal gland in the Chinese: a clinicopathologic study of 298 patients. Graefes Arch Clin Exp Ophthalmol. 2010;248(9):1345-9.

9. Woo KI, Yeom A, Esmaeli B. Management of Lacrimal Gland Carcinoma: lessons from the literature in the past 40 years. Ophthalmic Plast Reconstr Surg. 2016;32(1):1-10.

10. Henderson JW, Neault RW. En bloc removal of intrinsic neoplasms of the lacrimal gland. Am J Ophthalmol. 1976;82(6):905-9.

11. Esmaeli B, Golio D, Kies M, DeMonte F. Surgical management of locally advanced adenoid cystic carcinoma of the lacrimal gland. Ophthalmic Plast Reconstr Surg. 2006;22(5):366-70

12. Esmaeli B, Ahmadi MA, Youssef A, Diba R, Amato M, Myers JN, et al. Outcomes in patients with adenoid cystic carcinoma of the lacrimal gland. Ophthalmic Plast Reconstr Surg. 2004;20(1):22-6.

13. Pommier P, Liebsch NJ, Deschler DG, Lin DT, Mclntyre JF, Barker FG 2nd, et al. Proton beam radiation therapy for skull base adenoid cystic carcinoma. Arch Otolaryngol Head Neck Surg. 2006;132(11):1242-9.

14. Meldrum ML, Tse DT, Benedetto P. Neoadjuvant intracarotid chemotherapy for treatment of advanced adenocystic carcinoma of the lacrimal gland. Arch Ophthalmol. 1998;116(3):315-21.

15. Esmaeli B, Yin VT, Hanna EY, Kies MS, William WN Jr, Bell D, et al. Eye-sparing multidisciplinary approach for the management of lacrimal gland carcinoma. Head Neck. 2016;38(8):1258-62. 
16. Han J, Kim YD, Woo KI, Sobti D. Long-term outcomes of eye-sparing surgery for adenoid cystic carcinoma of lacrimal gland. Ophthalmic Plast Reconstr Surg. 2018;34(1):74-8.

17. Woo Kl, Sagiv O, Han J, Frank SJ, Kim YD, Esmaeli B. Eye-preserving surgery followed by adjuvant radiotherapy for lacrimal gland carcinoma: outcomes in 37 patients. Ophthalmic Plast Reconstr Surg. 2018;34(6):570-4.

18. Friedrich RE, Bleckmann V. Adenoid cystic carcinoma of salivary and lacrimal gland origin: localization, classification, clinical pathological correlation, treatment results and long-term follow-up control in 84 patients. Anticancer Res. 2003;23(2A):931-40.

19. Ni C, Cheng SC, Dryja TP, Cheng TY. Lacrimal gland tumors: a clinicopathological analysis of 160 cases. Int Ophthalmol Clin. 1982;22(1):99-120.

20. Tellado MV, McLean IW, Specht CS, Varga J. Adenoid cystic carcinomas of the lacrimal gland in childhood and adolescence. Ophthalmology. 1997; 104(10):1622-5.

21. Weis E, Rootman J, Joly TJ, Berean KW, Al-Katan HM, Pasternak S, et al. Epithelial lacrimal gland tumors: pathologic classification and current understanding. Arch Ophthalmol. 2009;127(8):1016-28.

22. Chawla B, Kashyap S, Sen S, Bajaj MS, Pushker N, Gupta K, et al. Clinicopathologic review of epithelial tumors of the lacrimal gland. Ophthalmic Plast Reconstr Surg. 2013;29(6):440-5.

23. Thariat J, Racadot S, Pointreau Y, Boisselier P, Grange JD, Graff P, et al. Intensitymodulated radiotherapy of head and neck cancers: dose effects on the ocular, orbital and eyelid structures. Cancer Radiother. 2016;20(6-7):467-74.

24. Marchand V, Dendale R. Normal tissue tolerance to external beam radiation therapy: eye structures. Cancer Radiother. 2010;14(4-5):277-83.

25. Brady LW. Ocular complications of high-dose radiotherapy. Oncology (Williston Park). 1996;10(7):981-2 4.

\section{Publisher's Note}

Springer Nature remains neutral with regard to jurisdictional claims in published maps and institutional affiliations.

Ready to submit your research? Choose BMC and benefit from:

- fast, convenient online submission

- thorough peer review by experienced researchers in your field

- rapid publication on acceptance

- support for research data, including large and complex data types

- gold Open Access which fosters wider collaboration and increased citations

- maximum visibility for your research: over $100 \mathrm{M}$ website views per year

At $\mathrm{BMC}$, research is always in progress.

Learn more biomedcentral.com/submissions 